\title{
An Improved Transformation and Optimized Sampling Scheme for the Numerical Evaluation of Singular and Near-Singular Potentials
}

\author{
Michael A. Khayat, Member, IEEE, Donald R. Wilton, Fellow, IEEE, \\ and Patrick W. Fink, Member, IEEE
}

\begin{abstract}
Simple and efficient numerical procedures using singularity cancellation methods are presented for evaluating singular and near-singular potential integrals. Four different transformations are compared and the advantages of the Radialangular transform are demonstrated. A method is then described for optimizing this integration scheme.
\end{abstract}

Index Terms - Numerical integration, Integral equations, Computation theory.

\section{INTRODUCTION}

$\mathrm{I}$ $\mathrm{T}$ is well known that singular potential integrals involved in the integral equations of electromagnetics often require special numerical considerations for their evaluation. Until recently, the method of singularity subtraction was used almost exclusively for the evaluation of singular and nearsingular terms. However, it becomes unwieldy and prone to error for bases of higher order, and it is not conducive to object-oriented programming practices, particularly in the context of multiple operators.

To extend the capabilities, accuracy, and maintainability of general-purpose codes, the subtraction method is being replaced in favor of purely numerical quadrature schemes. These schemes employ singularity cancellation methods in which a change of variables is chosen such that the Jacobian of the transformation cancels the singularity. Recently, a new singularity cancellation scheme, the arcsinh transformation, was presented for handling $1 / R$ singularities [1]. For singular integrals the method not only has several advantages over singularity subtraction methods, but also improves on some aspects of other singularity cancellation methods such as Polar [2] and Duffy [3] transformations. One drawback of the scheme, however, is its inability to efficiently calculate nearsingular integrals. To this end, we have extended the Duffy and polar transformations to handle near-singularities, and introduced a new scheme referred to as the Radial-angular transformation $[4,5]$. In this presentation we summarize and compare the four transformations for $1 / R$ type kernels and describe a method for optimizing the Radial-angular transformation.

\section{GREEN'S FUNCTIONS WITH 1/R SINGULARITIES}

\section{A. Theory}

In this section the arcsinh, extended Duffy, extended radial (or polar) and radial-angular transformations are compared. To evaluate potential integrals of the form

$$
\mathbf{I}=\int_{\mathrm{D}} \boldsymbol{\Lambda}\left(\mathbf{r}^{\prime}\right) \frac{e^{-j k R}}{4 \pi R} d \mathrm{D}
$$

where $R=\left|\mathbf{r}-\mathbf{r}^{\prime}\right|$ is the distance between an observation point at $\mathbf{r}$ and source points on triangular domains $\mathrm{D}$, a nearby observation point is projected onto a parent triangular element, as shown in Fig 1. The parent triangle is then split into three subtriangles about the projected observation point. The geometry of a typical subtriangle with origin at the projected observation point is shown in Fig. 2 .

The general form of the transformed integral over the subtriangle is given by

$$
\begin{aligned}
& \int_{0}^{h} \int_{y \cot \phi_{L}}^{y \cot \phi_{U}} h(x, y) d x d y \\
& \quad=\int_{v_{L}}^{v_{U}} \int_{u_{L}}^{u_{U}} h[x(u, v), y(u, v)] \mathrm{J}(u, v) d u d v
\end{aligned}
$$

where the function $h(x, y)$ is a product of the basis and Green's potential. Ideally, the transform's Jacobian J would exactly cancel the singular (static) part of the kernel, and the subtriangle would map into a rectangular domain such that repeated Gauss-Legendre integration of low order may be used. A summary of the transformations investigated is given in Table 1, followed by a synopsis of their actual properties.

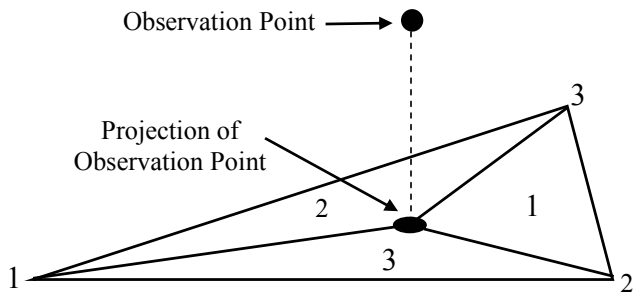

Fig. 1. Subdividing a triangle into subtriangles about the projected observation point. 


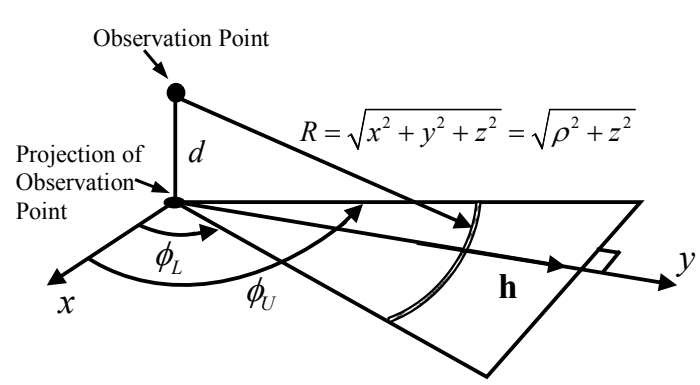

Fig. 2. Subtriangle geometry

TABLE 1:

SUMMARY OF TRANSFORMATIONS

\begin{tabular}{|c|c|c|c|}
\hline & TRANSFORMATION & $\mathrm{J}(\boldsymbol{u}, v)$ & INTEGRATION LIMITS \\
\hline Arcsinh & $\begin{array}{l}u=\sinh ^{-1} \frac{x}{\sqrt{y^{2}+z^{2}}} \\
v=\mathrm{y}\end{array}$ & $R$ & $\begin{array}{l}u_{\mathrm{L}, \mathrm{U}}=\sinh ^{-1}\left(\frac{y \cot \phi_{\mathrm{LU}}}{\sqrt{y^{2}+z^{2}}}\right) \\
v_{\mathrm{L}, \mathrm{U}}=0, h\end{array}$ \\
\hline Extended Duffy & $\begin{array}{l}u=\frac{x}{\sqrt{y^{2}+z^{2}}} \\
v=\mathrm{y}\end{array}$ & $\sqrt{y^{2}+z^{2}}$ & $\begin{array}{l}u_{\mathrm{L}, \mathrm{U}}=\frac{y \cot \phi_{\mathrm{L}, \mathrm{U}}}{\sqrt{y^{2}+z^{2}}} \\
v_{\mathrm{L}, \mathrm{U}}=0, h\end{array}$ \\
\hline Extended Radial (Polar) & $\begin{array}{l}u=\tan ^{-1} \frac{y}{x}=\phi \\
v=R\end{array}$ & $R$ & $\begin{array}{l}u_{\mathrm{L}, \mathrm{U}}=\phi_{\mathrm{L}, \mathrm{U}} \\
v_{\mathrm{L}, \mathrm{U}}=|z|, \sqrt{z^{2}+(h / \sin u)^{2}}\end{array}$ \\
\hline Radial-Angular & $\begin{array}{l}u=\ln \tan \frac{\phi}{2} \\
v=R\end{array}$ & $\frac{R}{\cosh u}$ & $\begin{array}{l}u_{\mathrm{L}, \mathrm{U}}=\ln \tan \frac{\phi_{\mathrm{L}, \mathrm{U}}}{2} \\
v_{\mathrm{L}, \mathrm{U}}=|z|, \sqrt{z^{2}+(h \cosh u)^{2}}\end{array}$ \\
\hline
\end{tabular}

arcsinh:

- Exact singularity cancellation

- Rectangular integration domain results only for $z=0$ and very sensitive to variations in $z$

- A single sample point integrates static kernel exactly for constant source densities for $z=0$

extended Duffy:

- Extends Duffy to the case $z \neq 0$

- Inexact singularity cancellation for any $z$

- A rectangular integration domain results only for $z=0$ and very sensitive variations in $z$

radial:

- Extends polar form to the case $z=0$

- Exact singularity cancellation

- Non-rectangular integration domain, but is insensitive to variations in $z$

radial-angular:

- Exact singularity cancellation

- Non-rectangular integration domain for $z=0$, but $\cosh u$ angular variation in Jacobian is compensated for by $v_{U}$ radial limit; insensitive to $z=0$ variation

- Single sample point integrates the static kernel exactly for constant source densities for $z=0$

For $z=0$, the arcsinh and radial-angular approaches are nearly identical. The radial-angular transformation presents the best compromise for both $z=0$ and $z \neq 0$ and, therefore, we will look at this method in more detail.

From Eq. 1 and Fig. 2 we have

$$
\begin{aligned}
\mathbf{I} & =\int_{\phi_{L}}^{\phi_{U}} \int_{0}^{h / \sin \phi} \boldsymbol{\Lambda}\left(\mathbf{r}^{\prime}\right) \frac{e^{-j k R}}{4 \pi R} \rho d \rho d \phi \\
& =\int_{\phi_{L}}^{\phi_{U}} \int_{R_{L}}^{R_{U}} \boldsymbol{\Lambda}\left(\mathbf{r}^{\prime}\right) \frac{e^{-j k R}}{4 \pi R} R d R d \phi,
\end{aligned}
$$

where $\left\{R_{L}, R_{U}\right\}=\left\{|z|, \sqrt{z^{2}+(h / \sin \phi)^{2}}\right\} . \quad$ For $\boldsymbol{\Lambda}\left(\mathbf{r}^{\prime}\right)=1$ and $k=0$, Eq. 3 becomes

$$
\mathbf{I}=\frac{1}{4 \pi} \int_{\phi_{L}}^{\phi_{U}}\left\{\sqrt{z^{2}+(h / \sin \phi)^{2}}-|z|\right\} d \phi
$$

If $h \square d$ then the kernel in Eq.4 is proportional to $h / \sin \phi$. In order to smooth the angular dependence we let

$$
d u=\frac{d \phi}{\sin \phi} .
$$

This yields

$$
\begin{aligned}
\mathbf{I} & =\int_{u_{L}}^{u_{U}} \int_{R_{L}}^{R_{U}} \boldsymbol{\Lambda}\left(\mathbf{r}^{\prime}\right) \frac{e^{-j k R}}{4 \pi \cosh u} d R d u \\
& =\mathrm{J} \sum_{k=(i, j)} W_{k} \Lambda\left(\mathbf{r}^{\prime}(k)\right) \frac{e^{-j k R^{(k)}}}{4 \pi R^{(k)}},
\end{aligned}
$$

where $u_{L, U}=\ln \left[\tan \left(\phi_{L, U} / 2\right)\right]$,

$$
W_{k}=\frac{w_{i} w_{j}\left[u_{U}-u_{L}\right]\left[\sqrt{z^{2}+h^{2} \cosh ^{2} u^{(j)}}-|z|\right] R^{(i, j)}}{\mathrm{J} \cosh u^{(j)}}
$$

are the weights, and $\mathrm{J}$ is the Jacobian of the triangle. Hence, the integral can be calculated using repeated Gauss-Legendre quadrate with weights $w_{(i, j)}$. The details of how to evaluate potential integrals in simplex coordinates is shown in [1] and, therefore, will not be repeated here.

\section{B. Results}

In this section we compare convergence results for a subtriangle with geometry defined as in Fig 3 . This geometry allows one to limit the degrees of freedom and create optimized sampling schemes, which are given in the following section.

For any given subtriangle one determines its maximum edge length. As Fig. 2 illustrates, this defines vertices 1 and 2 of the subtriangle with vertex 3 located between the circular arcs in Fig. 3. These arcs have radii equal to the maximum edge length and are centered on its end points; they define the locus 


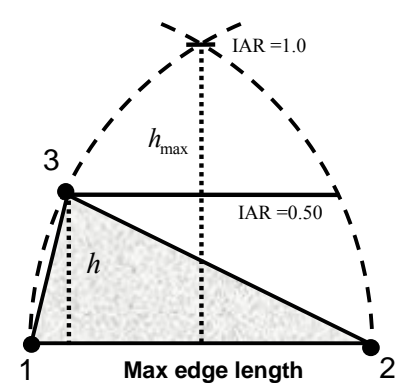

Fig. 3. Geometry of a subtriangle showing the Inverse Aspect Ratio

length and are centered on its end points; they define the locus of the third vertex whose attached edges are no longer than the maximum edge. In order to quantify the subtriangle geometry we introduce a quantity called the Inverse Aspect Ratio (IAR), which is defined as

$$
I A R=\frac{h}{h_{\max }}=\frac{2}{\sqrt{3}} \frac{h}{\ell_{\text {max }}},
$$

Where $\ell_{\text {max }}$ is the maximum edge length. The IAR is normalized such that $0 \leq \mathrm{IAR} \leq 1$. The maximum IAR occurs when vertex 3 is at the intersection of the two circles (resulting in an equilateral triangle), and the minimum occurs as vertex 3 approaches the maximum edge length.

The convergence of the potential due to a linear source density for a self term and near-self term are shown in Figs. 4 and 5, respectively. The reference values, shown in Table 2, were calculated using the arcsinh and radial-angular methods. The subtriangle was subdivided until the two transformations agreed to at least 11 significant digits. The maximum edge length was chosen to be $1 / 6 \lambda$, and a linear source density was defined to be unity at node 1 and zero along the opposite edge. Note that for self terms the arcsinh and radial-angular transformations have nearly identical convergence behavior.

For the extended radial and radial-angular methods the basis functions contain the terms $x=\rho \cos \phi$ and $y=\rho \sin \phi$, where $\rho=\sqrt{R^{2}-z^{2}}=\sqrt{R+z} \sqrt{R-z}$. For $z \neq 0$, the convergence curves flatten because of the $\sqrt{R-z}$ term. In order to integrate this term accurately a new quadrature scheme was developed based on the family of functions

$$
F=\{1, \sqrt{R-z}, R, R \sqrt{R-z}, \ldots\} .
$$

A partial table for this polynomial-root quadrature scheme is shown in Table 3. From Figs. 4 and 5 the radial-angular transformation has the best convergence of the four transformations studied. However, for $z=0$ Gauss-Legendre points should be used for the radial sampling, while the polynomial-root quadrature scheme should be used for $z \neq 0$.

\section{Optimization}

Based on the results in the previous section, we can now outline a scheme for optimizing the radial-angular transformation, the results of which are shown in Table 4. Numerical experiments have verified that the largest integration error occurs when the third vertex (i.e. singularity) lies along one of the bounding circular arcs, the $1 / R$ variation in the kernel has the largest variation as measured from the vertex to the opposite edge. Hence, if convergence is achieved for singularities lying along a circular arc, then convergence can be guaranteed for vertices along the same line parallel to the largest side, i.e. having the same IAR.

We define the scheme as optimized which has the fewest number of sample points used to achieve convergence given: 1. The IAR, 2. The distance $z$ from the source plane, 3. Self term or near-self term within the plane of the triangle, and 4. the desired accuracy chosen by the user. In this work we studied the range $I A R=\left[1 \times 10^{-8}, 1.0\right]$. We chose a maximum edge length of $1 / 6 \lambda$ for the parent triangle. Based on experience, we define a near-self term to be an observation point that lies within the region Ratio $\leq 1$, where Ratio $=\left|\mathbf{r}_{c}\right| / \ell_{\max }$ and $\left|\mathbf{r}_{c}\right|$ is the distance from the observation point to the centroid of the parent triangle. Therefore, the maximum edge length of a subtriangle can exceed $1 / 6 \lambda$. In order to place an upper bound on $\ell_{\max }$ for near-self terms we observe that if an observation point is lying on a bounding

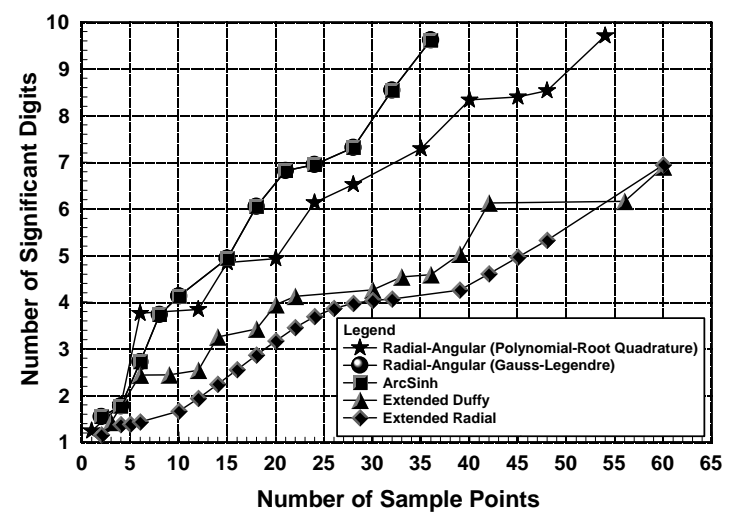

Fig. 4. Self term convergence for IAR $=0.1$.

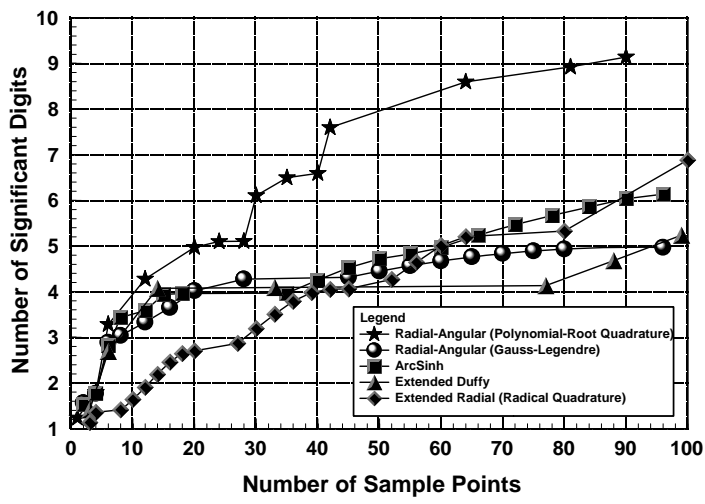

Fig. 5. Near-self term convergence for IAR $=0.1$ and $\mathrm{d}=1 \times 10^{-2} \lambda$.

TABLE 2:

REFERNCE POTENTIAL VALUES

\begin{tabular}{||c|c|c||}
\hline \hline$D$ & REAL & IMAGINARY \\
\hline 0.0 & 0.015220872777 & -0.0023610287866 \\
\hline $1 \times 10^{-2}$ & 0.015219642767 & -0.0023610286779 \\
\hline
\end{tabular}


TABLE 3:

RADICAL QUADRATURE POINTS

\begin{tabular}{||l|l|l||}
\hline \hline $\mathrm{N}$ & \multicolumn{1}{|c|}{ Nodes $X_{i}$} & \multicolumn{1}{|c|}{ Weights $W_{i}$} \\
\hline \multirow{3}{*}{2} & 0.12606123086601956 & 0.3639172365120473 \\
\cline { 2 - 3 } & 0.7139387691339825 & 0.6360827634879527 \\
\hline \multirow{3}{*}{3} & 0.045088504179695364 & 0.13965395980291434 \\
\cline { 2 - 3 } & 0.34872938419346483 & 0.45848221271917206 \\
\cline { 2 - 3 } & 0.8306719075452189 & 0.4018638274779136 \\
\hline \multirow{4}{*}{4} & 0.019532819681463730 & 0.06236194190019799 \\
\cline { 2 - 3 } & 0.17339692801497078 & 0.25969509521658130 \\
\cline { 2 - 3 } & 0.522956026924229700 & 0.40692913602039693 \\
\cline { 2 - 3 } & 0.88905249698491430 & 0.27101382686282377 \\
\hline \multirow{4}{*}{5} & 0.0097091631326854 & 0.0314958290411988 \\
\cline { 2 - 3 } & 0.0927420087986999 & 0.1478177401386180 \\
\cline { 2 - 3 } & 0.3158723139054450 & 0.2927739741663490 \\
\cline { 2 - 3 } & 0.6431824779012770 & 0.3343492761944000 \\
\cline { 2 - 3 } & 0.9219651106129180 & 0.1935631804594320 \\
\hline
\end{tabular}

TABLE 4:

4 SIGNIFICANT DIGIT SAMPLING SCHEMES FOR SUBTRIANGLE WITH MAXIMUM EDGE $=5 \lambda / 18$

\begin{tabular}{||c|c|c|}
\hline$d / \ell_{\max }$ & IAR & Rad $\times$ Trans \\
\hline \multirow{5}{*}{$<10^{-8}$} & {$[0.9,1.0]$} & $3 \times 3$ \\
\cline { 2 - 3 } & {$[0.4,0.9)$} & $3 \times 4$ \\
\cline { 2 - 3 } & {$[0.2,0.4)$} & $3 \times 5$ \\
\cline { 2 - 3 } & {$[0.1,0.2)$} & $3 \times 6$ \\
\cline { 2 - 3 } & {$\left[10^{-2}, 0.1\right)$} & $3 \times 7$ \\
\cline { 2 - 3 } & {$\left[10^{-3}, 10^{-2}\right)$} & $3 \times 8$ \\
\cline { 2 - 3 } & {$\left[10^{-4}, 10^{-3}\right)$} & $3 \times 10$ \\
\cline { 2 - 3 } & {$\left[10^{-5}, 10^{-4}\right)$} & $3 \times 11$ \\
\cline { 2 - 3 } & {$\left[10^{-6}, 10^{-5}\right)$} & $3 \times 12$ \\
\hline \multirow{5}{*}{$10^{-8}, 0.5$} & {$[0.9,1.0]$} & $4 \times 3$ \\
\cline { 2 - 3 } & {$[0.4,0.9)$} & $4 \times 4$ \\
\cline { 2 - 3 } & {$[0.2,0.4)$} & $4 \times 5$ \\
\cline { 2 - 3 } & {$[0.1,0.2)$} & $4 \times 6$ \\
\cline { 2 - 3 } & {$\left[10^{-2}, 0.1\right)$} & $4 \times 7$ \\
\cline { 2 - 3 } & {$\left[10^{-3}, 10^{-2}\right)$} & $4 \times 10$ \\
\cline { 2 - 3 } & {$\left[10^{-4}, 10^{-3}\right)$} & $4 \times 12$ \\
\cline { 2 - 3 } & {$\left[10^{-5}, 10^{-4}\right)$} & $4 \times 15$ \\
\cline { 2 - 3 } & {$\left[10^{-6}, 10^{-5}\right)$} & $4 \times 17$ \\
\hline$[0.5,1.5)$ & {$\left[10^{-6}, 1.0\right]$} & 16 pt. Gauss \\
\hline$\geq 1.5$ & {$\left[10^{-6}, 1.0\right]$} & 7 pt. Gauss \\
\hline
\end{tabular}

circular arc and $I A R \rightarrow 0$, the centroid of a triangle will cross the edge a distance $\ell_{\max } / 3$ from a vertex. Hence, the maximum edge length achieved for a near-self term is given by $\ell_{\text {max,near }}=\ell_{\text {max,self }}($ Ratio $+2 / 3)$. Choosing Ratio $=1$ and $\ell_{\text {max,self }}=1 / 6 \lambda$ we obtain $\ell_{\text {max,naar }}=5 / 18 \lambda$.

An additional variable studied for optimization was the basis function. For a given observation point, an optimized sampling scheme was chosen such that convergence was achieved for four different basis functions: a constant function and three linear functions. The three linear functions represent the bases defined at each of the three vertices. In order to justify analyzing each integral independently, as opposed to a linear combination, consider the error in computing each one as defined by $I_{i}-\tilde{I}_{i}=\varepsilon_{i} I_{i}$, where $\varepsilon_{i}$ is the relative error in computing the integral. If we define the relative error of computing a linear combination of integrals as

$$
\bar{\varepsilon}_{N}=\frac{\sum_{i=1}^{N} a_{i} \varepsilon_{i} I_{i}}{\sum_{i=1}^{N}\left|a_{i}\right|\left|I_{i}\right|},
$$

then we have by the triangle inequality

$$
\left|\bar{\varepsilon}_{N}\right| \leq \max \left|\varepsilon_{i}\right| \text {. }
$$

Defined in this way, we have the simple result that the relative error of a linear combination of $\mathrm{N}$ terms, each with error $\left|\varepsilon_{i}\right| \leq \varepsilon=\max \left|\varepsilon_{i}\right|$, suffers no loss of significant digits.

Table 4 shows the optimized sampling schemes of near-self terms for 4 significant digits. It was determined that the sampling schemes for self and near-self terms were similar enough that the near-self schemes could be used for both cases without a significant loss in efficiency. Note that the optimized sampling schemes are applied to a cylindrical, pill box region around the subtriangle. The curved brackets in the tables represents an open boundary, while the square bracket represents a closed boundary.

\section{CONCLUSION}

Accurate methods for the purely numerical evaluation of singular and near-singular potentials with $1 / R$ singularities have been presented. Of the four transformations studied, the radial-angular transformation had the best overall convergence. An optimization scheme was developed for this transformation based on the observation point location and the desired number of significant digits.

\section{REFERENCES}

[1] M. A. Khayat and D. R. Wilton, "Numerical Evaluation of Singular and Near-Singular Potential Integrals," IEEE Trans. Antennas Propagat.., vol. 53, no. 10, pp. 31803190, Oct. 2005.

[2] M. G. Duffy, "Quadrature over a pyramid or cube of integrands with a singularity at a vertex," SIAM J. Num. Anal., vol. 19, issue 6, pp. 1260-1262, 1982.

[3] L. Rossi and P. J. Cullen, "On the fully numerical evaluation of the linear-shape function times the 3-D Green's function on a plane triangle," IEEE Trans. Microwave Theory Tech., Vol. 47, pp. 398-402, April 1999.

[4] P. W. Fink, D. R. Wilton, and M. A. Khayat, "Issues and Methods Concerning the Evaluation of Hypersingular and Near-Hypersingular Integrals in BEM Formulations", Proc. ICEAA, pp. 861-864, Torino, Italy, 2005.

[5] P.W. Fink, D. R. Wilton, and M. Khayat, "Refinement of Methods for Evaluation of Near-Hypersingular Integrals in BEM Formulations", URSI National Radio Science Meeting, Albuquerque, N.M., 2006. 